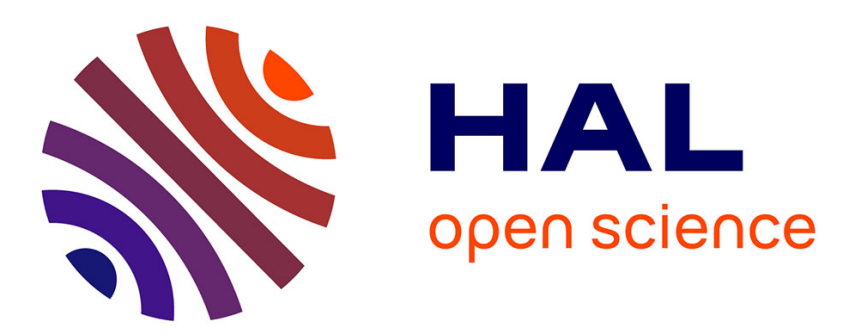

\title{
The step free-energy and the behaviour at the roughening transition of lattice gauge theories
}

\author{
J.M. Drouffe, J.M. Luck, J.B. Zuber
}

\section{To cite this version:}

J.M. Drouffe, J.M. Luck, J.B. Zuber. The step free-energy and the behaviour at the roughening transition of lattice gauge theories. Journal de Physique, 1981, 42 (5), pp.653-656. 10.1051/jphys:01981004205065300 . jpa-00209051

\section{HAL Id: jpa-00209051 https://hal.science/jpa-00209051}

Submitted on 1 Jan 1981

HAL is a multi-disciplinary open access archive for the deposit and dissemination of scientific research documents, whether they are published or not. The documents may come from teaching and research institutions in France or abroad, or from public or private research centers.
L'archive ouverte pluridisciplinaire HAL, est destinée au dépôt et à la diffusion de documents scientifiques de niveau recherche, publiés ou non, émanant des établissements d'enseignement et de recherche français ou étrangers, des laboratoires publics ou privés. 


\title{
The step free-energy and the behaviour at the roughening transition of lattice gauge theories
}

\author{
J. M. Drouffe, J. M. Luck and J. B. Zuber \\ DPh-T, CEA Saclay, 91191 Gif sur Yvette Cedex, France
}

(Reçu le 29 décembre 1980, accepté le 28 janvier 1981)

\begin{abstract}
Résumé. - L'énergie libre de marche est le coût nécessaire à la création d'une marche sur une boucle de Wilson plate. Nous avons calculé cette quantité par unité de longueur au $8 \mathrm{e}$ ordre dans le développement de couplage fort des théories de jauge $Z_{2}$ sur réseau à 3 et 4 dimensions. Nous avons analysé la façon dont elle s'annule à la transition rugueuse. Nos résultats sont compatibles avec une singularité essentielle exp $-C\left(t_{\mathrm{R}}-t\right)^{-1 / 2}$ suggérée par la relation avec le modèle $X Y$.
\end{abstract}

Abstract. - The step free-energy is the cost in free energy to create a single step in a planar Wilson loop. We compute it, per unit length, to eighth order in a strong coupling expansion of 3-D and 4-D $Z_{2}$ gauge theories, and analyse its vanishing at the roughening transition. Our results are consistent with an essential singularity $\exp -C\left(t_{\mathrm{R}}-t\right)^{-1 / 2}$, expected from a relationship with the $X Y$-model.

1. Introduction. - It has recently been realized that lattice gauge theories experience a roughening transition [1-4]. Because of long range fluctuations of large two-dimensional surfaces, physical quantities attached to such surfaces, such as the string tension, have a singularity at a finite value of the coupling. The first works devoted to this roughening phenomenon in lattice gauge theories have provided ample evidence for the existence of this singularity for arbitrary gauge groups and low (space-time) dimensions $3 \leqslant d \leqslant 5$. In this paper we address the problem of finding an effective theory in the roughening region. In three dimensions, it is well known [5-6] that the $Z_{2}$ lattice gauge theory (dual to the Ising model) is, near the roughening coupling, equivalent to a $X Y$-model. The low coupling (rough) phase of the former corresponds to the low-temperature (no free vortices) phase of the latter. For instance, the step free-energy $f$, which is the free energy per unit length associated to the creation of a single step in the interface, is proportional to the inverse correlation length $\xi^{-1}$ of the $X Y$-model [7]. It vanishes in the rough phase, and approaches zero as [8]

$$
f \sim \xi^{-1} \sim \exp -\frac{C}{\sqrt{\beta_{\mathrm{r}}-\beta}}
$$

as $\beta=1 / g_{0}^{2}$ approaches $\beta_{\mathrm{r}}$ from below.

Strictly speaking, this equivalence between the roughening and $X Y$ transitions holds only for the so-called solid-on-solid (S.O.S.) version of the $Z_{2}$ model [6], where disconnected parts and " overhang " configurations are discarded. However, we do not expect this modification to affect the behaviour (1), since the neglected configurations are not yet numerous and do not seem to play an important role for $\beta \simeq \beta_{r}$. Also, the same singular behaviour (1) is likely to arise in other three-dimensional gauge theories, based on different groups. The reason is again that, at the roughening transition, group-dependent configurations play a very minor role. This is assessed in particular by the apparent universality of the location of the roughening coupling, when measured in a suitable scale [1-3].

In four dimensions, on the other hand, no such simple equivalence exists. It has been argued however [1] that an effective theory might be given by two decoupled $X Y$-models. If correct, this would lead to the same behaviour (1) for the step free-energy, and conversely evidence for such a singular behaviour would support this argument. In this paper, we try to find such an evidence from strong coupling expansions of $f$. We restrict ourselves to the $Z_{2}$ group.

In section 2, we explain how this calculation may be performed, while section 3 deals with the analysis of the series. As we shall see, these series are difficult to analyse, and we shall present our tentative conclusions in the last section. A similar analysis has been reported by Pearson [4] using a four term expansion within the Hamiltonian formulation. 
2. Strong coupling expansion of the step energy. To define the step free-energy $f$ in a d-dimensional $Z_{2}$ lattice gauge theory, we introduce a Wilson loop along a contour with a single step, as shown in figure 1 . The expectation value of such a loop of area $A$ (with the usual Boltzmann factor $\exp \beta \sum_{\text {plaquettes }} U_{\mathrm{P}}$ ) has the asymptotic behaviour

$$
\langle W\rangle=\exp (-k A-L f)
$$

where $k$ is the string tension and $f$ the step free-energy.

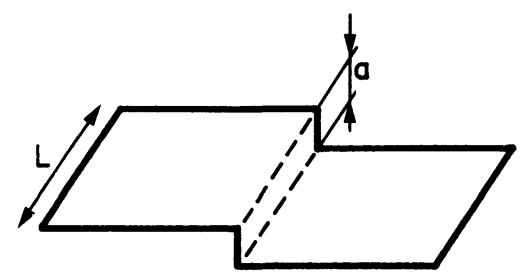

Fig. 1. - Configuration of the Wilson loop for the definition of the step free-energy. The step is one lattice spacing high.

Unfortunately, its strong coupling (small $\beta$ ) expansion does not exhibit this behaviour : the large $L$ limit does not commute with the expansion. Typically taking into account the first correction to $\langle W\rangle$, coming from diagrams of figure 2 , leads to

$$
\langle W\rangle=t^{A+L}\left[1+L(L+1) t^{2}+\cdots\right]
$$

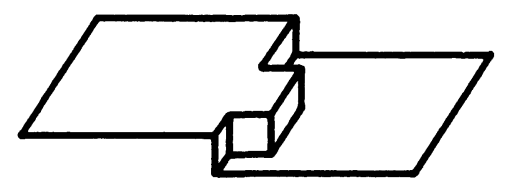

Fig. 2. - First corrections to the diagram of figure 1, proportional to $\frac{L(L+1)}{2}$.

where

$$
t=\tanh \beta
$$

and this expression cannot be cast into the form (2).

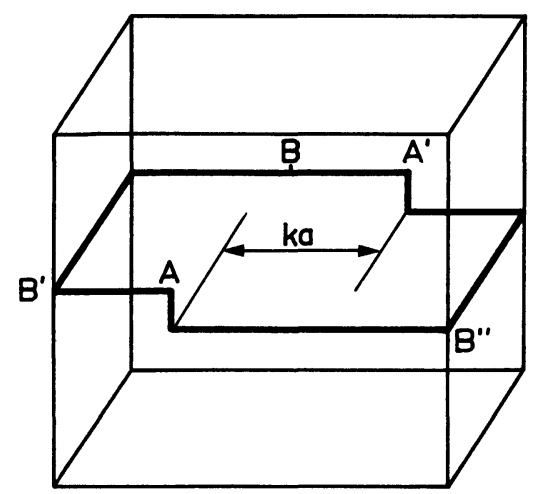

Fig. 3. - Shifted loop for the actual calculation of $\langle W\rangle$. Skew periodic boundary conditions identify $\mathrm{A}$ and $\mathrm{A}^{\prime}, \mathrm{B}, \mathrm{B}^{\prime}$ and $\mathrm{B}^{\prime \prime}$.
This desease is well known in high temperature calculations of correlation lengths in spin models [9], and the cure will be the same. Instead of calculating $\langle W\rangle$ for the loop of figure 1 , we consider rather the family of loops depicted on figure 3 ; the step on opposite edges of the loop has been shifted by an integer multiple of the lattice spacing $k . a(k \gtrless 0)$.

Furthermore, skew periodic boundary conditions are introduced, i.e. points $\mathrm{A}$ and $\mathrm{A}^{\prime}$ are identified, etc... For each $k,\langle W\rangle$ has again the form (1), but now the strong coupling expansion of $\langle W\rangle$, averaged on all the shifted configurations, is consistent with (1).

From this point on, the computation proceeds through the usual diagrammatic expansion, and we shall not bother the reader with its description.

In 3 and 4 dimensions, the expansion of $f$ to order eight reads :

$$
\begin{aligned}
& d=3 ;-f=\ln t+2 t+\frac{2}{3} t^{3}+ \\
& +\frac{2}{5} t^{5}-t^{6}-\frac{12}{7} t^{7}-4 t^{8} \\
& d=4 ;-j=\ln t+2 t+\frac{2}{3} t^{3}+2 t^{4}+ \\
& +\frac{22}{5} t^{5}+3 t^{6}+\frac{128}{7} t^{7}+14 t^{8}
\end{aligned}
$$

In three dimensions, we can also do the same calculation for the S.O.S. version of the model. It turns out that only the last term of $(5 a)$ is affected and changed into $-6 t^{8}$. In practice, this modification will not change the forthcoming results. Finally, we notice that, as expected, the first terms of these expansions reproduce the two-dimensional Ising boundary free energy $[10]: \ln [t(1+t) /(1-t)]$.

3. Analysis of the roughening singularity. - We now turn to the analysis of the singularity of the step free-energy $f$ at the roughening coupling $t_{\mathrm{R}}$. Along with the expansions (5) we may consider the expansion of $E=t \frac{\partial f}{\partial t}$, the step energy, which is also supposed to vanish at $t_{\mathrm{R}}$. We recall that previous analyses $[1-3,6]$ yield $t_{\mathrm{R}} \simeq 0.46$ at $d=3, t_{\mathrm{R}} \simeq 0.40$ at $d=4$.

As will become clear in the following, the expansions (5) are still much too short to yield unambiguous results. The best we can do is to rule out some possibilities and make suggestions.

First, we have checked that the expansions (5) of $f$ or the corresponding expansions of $E$ are inconsistent with an algebraic singularity $\left(t_{\mathrm{R}}-t\right)^{\alpha}$. Indeed, calculating the expansion of the logarithmic derivatives of $(-f-\ln t)$ or of $E$, and looking at the poles of their Padé extrapolations yields very unstable results. Also there is not yet any apparent sign of divergence of $f^{-1}$ or of $E^{-1}$ at $t_{\mathrm{R}}$. This also prevents us from comparing the expansion of $E_{\text {S.o.s. }}$ (or its Padé approximants) to the Monte-Carlo data of reference [11]. All these negative results may be interpreted as signs that the singularity of $f$ and $E$ is weak. 
We then turn to the Ansatz

$$
\begin{aligned}
& -f \sim\left(t_{\mathrm{R}}-t\right)^{\beta} \exp -C\left(t_{\mathrm{R}}-t\right)^{\alpha} \\
& -E \sim\left(t_{\mathrm{R}}-t\right)^{\beta-1+\alpha} \exp -C\left(t_{\mathrm{R}}-t\right)^{\alpha}
\end{aligned}
$$

where we allow the possibility of an algebraic singularity on top of an essential singularity. We have tried several methods to test this assumption.

1) Padé extrapolations of the logarithmic derivative of $\ln E$ are again rather unstable and seem more sensitive to a spurious "antiferromagnetic » coupling $t_{\mathrm{R}} \simeq-0.45$, reminiscent of the zero at $-\sqrt{2}+1$ of the two-dimensional Ising boundary energy

$$
E=1+\frac{2 t}{1-t^{2}} \quad[10]
$$

Only the last approximant at $d=4$ has a pole at $t_{\mathrm{R}}=0.412$ with residue $\alpha=-0.53$. It would be nice to check whether this result persists to higher orders.

2) A refined ratio method, advocated by Parisi [12], fails here because of the strong oscillations of the series (5).

3) Finally, the dominant term $\ln t$ in $f$ suggests us to use an alternative method to test the singularity (6). If $f$ vanishes at $t_{\mathrm{R}}$ as indicated in (6), so does $1-\mathrm{e}^{-f}$. Then $\varphi=\ln \left(1-\mathrm{e}^{-f}\right)$, which has a power series expansion, should have an algebraic singularity. This is what we find, using three different methods of analysis.

i) Computing the ratios of successive terms in the series of $\varphi$ yields

$$
\begin{aligned}
& t_{\mathrm{R}} \sim 0.47-0.48 \quad \text { at } \quad d=3 \\
& t_{\mathrm{R}} \sim 0.41-0.42 \quad \text { at } \quad d=4
\end{aligned}
$$

in rather good agreement (though a little too high) with other estimates [1-3, 6].

ii) The logarithmic derivative of $\varphi$ has the poles and residues listed in table $I$. The location of the singularity is again correctly reproduced, while the residue at $d=3$ seems closer to $-2 / 3$ than to $-1 / 2$, the value expected from the relationship with the $X Y$-model. This is no surprise since experience tells us that residues are generally not very well reproduced

Table I. - Nearest real pole and residue of the successive Padé approximants of the logarithmic derivative of $\varphi=\ln \left(1-\mathrm{e}^{-f}\right)$ at $d=3$ and $d=4$.

\begin{tabular}{|c|c|r|r|c|}
\hline & {$[2 / 3]$} & {$[3 / 3]$} & {$[3 / 4]$} & {$[4 / 4]$} \\
\hline$d=3$ & $t_{\mathrm{R}}=0.454$ & 0.460 & 0.459 & 0.460 \\
& $\alpha=-0.643$ & -0.680 & -0.675 & -0.681 \\
\hline \multirow{2}{*}{$d=4$} & $t_{\mathrm{R}}=0.367$ & 0.350 & 0.396 & 0.392 \\
& $\alpha=-0.397$ & -0.338 & -0.537 & -0.505 \\
\hline
\end{tabular}

to such a low order. On the other hand, the residue at $d=4$ is not inconsistent with $-1 / 2$.

iii) We have finally used the Padé-Mellin method on $\varphi$ [13]. We recall that in this method, the critical indices $\gamma_{\mathrm{i}}$ of a function $\varphi(t)$ at a given point $t_{\mathrm{R}}$ :

$$
\gamma \sim \Sigma c_{\mathrm{i}}\left(t_{\mathrm{R}}-t\right)^{-\gamma_{\mathrm{i}}}
$$

are identified as the poles of the transformed function $\mu(\varphi)$. The latter, whose series expansion is known from that of $\varphi$, is then analysed through Padé approximants. This method is very efficient to disentangle several confluent singularities at the same point. Unfortunately, it relies on the knowledge of $t_{\mathrm{R}}$. In our case, varying the order of the approximant and the location of $t_{\mathrm{R}}$ over the intervals $0.45 \leqslant t_{\mathrm{R}} \leqslant 0.47$ at $d=3,0.37 \leqslant t_{\mathrm{R}} \leqslant 0.41$ at $d=4$ has produced indices for the dominant singularity ranging between -0.5 and -0.67 at $d=3,-0.36$ and -0.67 at $d=4$. Checking that the indices of the derivative are shifted by one unit has given little further constraint.

It would have been nice to check either of these methods in a case which is known to have the behaviour (1) and where the expansion may be pushed to a higher order. This is indeed the case of the exactly solvable S.O.S. model on a body-centred cubic lattice considered by van Beijeren [14]. An equivalence with the 6-vertex model enables one to write the exact expression of $E$ :

$$
\begin{aligned}
E=t & \frac{\partial f}{\partial t}=\text { const. }\left(t_{\mathrm{R}}-t\right)^{-1 / 2} \times \\
& \times\left\{\frac{1}{2}+2 \sum_{n=1}^{\infty}(-1)^{n-1} n(\tanh n \lambda-1)\right\}
\end{aligned}
$$

where

$$
\lambda=-\ln t+2 \ln \left[\frac{1}{2}+\left(t_{\mathrm{R}}-t\right)^{1 / 2}\right] .
$$

The roughening coupling corresponds to $\lambda_{R}=0$, $t_{\mathrm{R}}=1 / 4$ and $E$ behaves at this point as :

$$
E \sim\left(t_{\mathrm{R}}-t\right)^{-3 / 2} \exp -\frac{\pi^{2}}{8\left(t_{\mathrm{R}}-t\right)^{1 / 2}}
$$

This model seems a perfect candidate to check the previous methods. Unfortunately, the essential singularity at $\lambda=0, t=t_{\mathrm{R}}$ is accompanied by a natural boundary along the imaginary axis in the $\lambda$ plane, as is clear on equation (7). As a consequence, the analytic structure in the $t$-plane near $t_{\mathrm{R}}=1 / 4$ is more complicated than a single branch point. When the previous methods of analysis are applied to the expansion, this reflects on a rough verification that $t_{\mathrm{R}}=0.25$ and very broad instabilities on the value of the critical index. Clearly, the best we can hope is that this natural boundary is not a universal feature, while the behaviour (1) is. 
4. Conclusion. - It must be clear to the reader that our conclusions cannot be very firm. We believe that we have ruled out the possibility that $f$ or $E$ vanish algebraically as $\left(t_{\mathrm{R}}-t\right)^{\alpha}$. We have found some evidence for an essential singularity $\exp -C\left(t_{\mathrm{R}}-t\right)^{x}$, with $1 / 2 \leqslant \alpha \leqslant 2 / 3$ at $d=3,1 / 3 \leqslant-\alpha \leqslant 2 / 3$ at $d=4$, therefore not inconsistent with our theoretical prejudice (1). Similar conclusions were reached by Kogut et al. [4]. It must also be recalled that at $d=3$, the analysis of the critical behaviour at the roughening point of the successive moments $\left\langle z^{2}\right\rangle,\left\langle z^{4}\right\rangle, \ldots$ of the distribution of heights of the interface from their series expansions has encountered some difficulties [15]. The values found for the indices disagree with those expected from universality [16], and may be even inconsistent with Schwartz inequality : $\left\langle z^{2}\right\rangle^{2} \leqslant\left\langle z^{4}\right\rangle$.

Much longer series should therefore be computed before a definite conclusion may be reached. We find it gratifying, however, to have found no violent disagreement with the theoretical prediction (1) and to have therefore some support to the relationship with the $X Y$-model.

Acknowledgments. - It is a pleasure to thank C. Itzykson, P. Moussa and J. Zinn-Justin for helpful suggestions and discussions.

\section{References}

[1] Itzykson, C., Peskin, M., Zuber, J. B., Phys. Lett. 95B (1980) 259.

Drouffe, J. M. and Zuber, J. B., Saclay preprints, DPhT/80/120 and 128, to appear in Nucl. Phys. B.

[2] Hasenfratz, A., Hasenfratz, E. and Hasenfratz, P., CERN Preprint Th. 2890.

[3] Lüscher, M., Münster, G. and Weisz, P., Nucl. Phys. B 180 [FS2] (1981) 1.

LÜsCHER, M., Desy preprint 80/87, to appear in Nucl. Phys. B. Münster, G. and WeIsZ, P., Nucl. Phys. B 180 [FS2] (1981) 13 and Desy preprint $80 / 97$

[4] Kogut, J. B., Pearson, R. P. and Shigemitsu, J., Illinois preprint I 11-TH-80-41.

Kogut, J., Pearson, R. P., Richardson, J., Shigemitsu, J. and Sinclatr, D., reported by R. P. Pearson in SantaBarbara preprint NSF-ITP-80-49.

[5] Chiu, S. T. and Weeks, J. D., Phys. Rev. B 14 (1976) 4978. José, J. V., Kadanoff, L. P., Kirkpatrick, S. and Nelson, D. R., Phys. Rev. B 16 (1977) 1217.

KNOPS, H. J. F., Phys. Rev. Lett. 39 (1977) 776.
[6] For a review and further references, see for instance Weeks, J. D. and Gilmer, G. H., Adv. Chem. Phys., Vol. 40, Ed. Prigogine and Rice (J. Wiley \& Sons) 1979.

[7] Swendsen, R. H., Phys. Rev. B 15 (1977) 5421.

[8] Kosterlitz, J. M. and Thouless, D. J., J. Phys. C 6 (1973) 1181.

Kosterlitz, J. M., J. Phys. C 7 (1974) 1046.

[9] Fisher, M. E. and Burford, R. J., Phys. Rev. 156 (1967) 583.

[10] ONSager, L., Phys. Rev. 65 (1944) 117.

[11] Leamy, H. J. and Gilmer, G. H., J. Crystal-Growth 24/25 (1974) 499

[12] Parisi, G., Phys. Lett. 90B (1980) 111.

[13] Baker JR., G. A. and Hunter, D. L., Phys. Rev. B 7 (1973) 3377.

Bessis, D., Moussa, P. and Turchetti, G., J. Phys. A 13 (1980) 2763.

[14] Van BeiJeren, H., Phys. Rev. Lett. 38 (1977) 993.

[15] Weeks, J. D., Gilmer, G. H. and Leamy, H. J., Phys. Rev. Lett. 31 (1973) 549.

[16] Оhта, T. and Kawasaki, K., Progr. Theor. Phys. 60 (1978) 365 and LUCK, J. M., to appear. 